\title{
Ecological Interface Design of a Tactical Airborne Separation Assistance Tool
}

\author{
Stijn B. J. Van Dam, Max Mulder, and M. M. van Paassen
}

\begin{abstract}
In a free-flight airspace environment, pilots have more freedom to choose user-preferred trajectories. An onboard pilot support system is needed that exploits travel freedom while maintaining spatial separation with other traffic. Ecological interface design is used to design an interface tool that assists pilots with the tactical planning of efficient conflict-free trajectories toward their destination. Desired pilot actions emerge from the visualization of workspace affordances in terms of a suitable description of aircraft (loco)motion. Traditional models and descriptions for aircraft motion cannot be applied efficiently for this purpose. Through functional modeling, more suitable locomotion models for trajectory planning are analyzed. As a result, a novel interface, the state vector envelope, is presented that is intended to provide the pilot with both low-level information, allowing direct action, and high-level information, allowing conflict understanding and situation awareness.
\end{abstract}

Index Terms-Ecological interface design (EID), functional modeling, navigation interface, separation assistance.

\section{NOMENCLATURE}

ASAS Airborne separation assurance system.

AH Abstraction hierarchy.

ATP Airborne trajectory planning.

CPA Closest point of approach.

EID Ecological interface design.

FBZ Forbidden beam zone.

HTF Heading travel function.

ND Navigation display.

PZ Protected zone.

SHTF Speed-heading travel function.

SVE State vector envelope.

\section{INTRODUCTION}

I N THE traditional airspace environment, capacity problems are expected in the near future due to growing air traffic. New concepts for air traffic management, such as free flight, permit a more flexible use of airspace with airborne determination of user-preferred trajectories that allow direct routing and cruise climb [16]. This will increase airspace capacity and reduce congestion problems, but at the same time, it leads to more complex traffic flows, increasing workload of air traffic controllers.

Manuscript received November 25, 2005; revised July 24, 2006, April 27, 2007, and November 20, 2007. Current version published October 20, 2008. This paper was recommended by Associate Editor E. Bass.

The authors are with the Faculty of Aerospace Engineering, Delft University of Technology, 2600 Delft, The Netherlands.

Digital Object Identifier 10.1109/TSMCA.2008.2001069
A possible way to reduce workload would be to delegate the separation task to the pilot. In order to assist pilots in self-separation, airborne separation assurance systems (ASAS) [6], like predictive ASAS (P-ASAS) [9], have been developed. ASAS systems form a strategic complement to currently deployed airborne collision avoidance systems (ACAS) like the traffic alert and collision avoidance system, TCAS II [17]. In the same way as ACAS systems do, traditional ASAS systems present a ready-to-use avoidance maneuver as a solution.

Generally, these automated systems present a limited set of explicit solutions to the pilot and have proven to be effective as far as providing conflict ${ }^{1}$ resolution and reducing workload are concerned. A few observations can be made, however, with respect to automated airborne self-separation support. First, when a conflict situation exists, explicit automated solutions hold pilots back from exploring solutions other than the one(s) presented and, therefore, may preclude the full exploitation of travel freedom and airspace capacity offered by future airspace environments. Second, in a complex traffic environment, nonroutine situations may arise, often beyond the scope of the automation and not anticipated for in the automation design. In these exceptional cases, the pilot's ability to improvise outperforms automated solutions. To support pilots in these unforseen situations, automation and instrumentation need to promote a high level of situation awareness.

These considerations call for an alternative approach to designing a system that assists pilots in maintaining separation. In this paper, the term "separation assistance" rather than "separation assurance" is used to label systems that help the pilot maneuver tactically in order to manage conflict situations, without giving an explicit resolution. Our objective is to show how ecological interface design (EID) [25] can be used to design such a decision-support tool. The design of this tool is based on an analysis of aircraft motion in the context of exploring travel possibilities. With the help of functional modeling techniques [13], aircraft behavior can be modeled in such a way that a presentation of the "travel function" allows pilots to directly perceive which control actions lead to a desired aircraft behavior, in a goal-directed fashion.

This paper is structured as follows. After some introductory definitions, first, the cognitive work associated with planning a conflict-free trajectory is analyzed. Then, two aircraft locomotion models and the visualizations and afforded planning strategies associated with them are discussed. The most promising interface, the SVE, is described, focusing on how it supports

\footnotetext{
${ }^{1}$ The term "conflict" means a predicted loss of separation between two aircraft in the near future. It will be defined more accurately below.
} 


\begin{tabular}{|c|c|c|c|}
\hline $\begin{array}{c}\text { main } \\
\text { purpose }\end{array}$ & $\begin{array}{c}\text { safe, productive and efficient } \\
\text { airspace travel }\end{array}$ & \multicolumn{2}{|c|}{$\begin{array}{c}\text { efficient and productive } \\
\text { conflict resolution and prevention } \\
\text { during cruise flight in horizontal plane }\end{array}$} \\
\hline $\begin{array}{l}\text { abstract } \\
\text { "key" } \\
\text { functions }\end{array}$ & \multicolumn{3}{|c|}{$\begin{array}{l}\text { spatial separation destination approximation } \\
\text { path deviation minimization implicit coordination }\end{array}$} \\
\hline $\begin{array}{l}\text { generalized } \\
\text { functions }\end{array}$ & \multicolumn{3}{|c|}{$\begin{array}{l}\text { aircraft (loco)motion } \\
\text { path control }\end{array}$} \\
\hline $\begin{array}{l}\text { physical } \\
\text { functions }\end{array}$ & & $\begin{array}{l}\text { lift, drag, visibility,... } \\
\text { destination position, ETA } \\
\text { aircraft information } \\
\text { terrain geography }\end{array}$ & $\begin{array}{l}\text { state info other a/c } \\
\text { lift } \\
\text { thrust } \\
\text { own aircraft state }\end{array}$ \\
\hline $\begin{array}{l}\text { physical } \\
\text { form }\end{array}$ & & $\begin{array}{l}\text { air, wind, weather } \\
\text { destination } \\
\text { aircraft } \\
\text { terrain }\end{array}$ & $\begin{array}{l}\text { ADS-B } \\
\text { wings } \\
\text { motor } \\
\text { sensors }\end{array}$ \\
\hline
\end{tabular}

Fig. 1. AH for ATP workspace.

pilot cognitive control. Finally, the results of a preliminary pilot evaluation are described.

\section{ECOlogical Interface Design}

EID is a design paradigm that addresses the cognitive interaction between humans and complex sociotechnical systems. It was originally applied to process control [25]. Its approach to interface design gives priority to the worker's environment ("ecology"), focusing on how the environment imposes constraints on the worker. EID supports worker adaptation and has proven better problem-solving performance when compared to other approaches [23].

EID consists of two main steps. The first step relates to the "content" and "structure" of the work domain, whereas the second addresses the interface "form." First, a workspace analysis tries to identify functionalities, constraints, and meansend relationships within the worker's environment, as these shape the possibilities of goal-directed worker actions within that environment. The identification is done using Rasmussen's abstraction hierarchy (AH) [15]. Second, EID aims to make these workspace constraints and means-end relationships easily visible on the display. It intends to express them in a meaningful functional way, taking advantage of the human capacity to directly perceive and act upon what the environment affords [7]. System functionalities and mechanisms that are often "hidden" from operators in traditional automated systems are made more transparent. In the present context, automation is used for the benefit of pilot situation awareness.

\section{Workspace ANALYSIS}

The analysis is made for the tactical navigation work, which will be referred to as airborne trajectory planning ${ }^{2}$ (ATP). The work involves onboard (re-)planning to achieve a safe,

\footnotetext{
${ }^{2}$ Note that the term "trajectory planning" might suggest onboard strategic flight management system trajectory planning, which it is not. The terms "ATP" or simply "planning" or "planning work" used throughout this paper strictly address onboard tactical trajectory planning work.
}

conflict-free, and efficient trajectory to the destination within a future free-flight-like airspace environment. A number of pilot-aircraft activities relate to this work, such as resolving and preventing conflicts, arriving on time at a destination, etc. The AH serves as a tool to set out a guide map of how different processes on different levels of functional abstraction relate with each other [25].

\section{A. Abstraction Hierarchy}

Fig. 1 shows an AH for ATP showing only the most important functions. Relations between functions are not explicitly shown. The AH is a stratified hierarchical description of the workspace, defined by means-end relationships between adjacent levels [25]. In the vertical direction, a functional "means-end" decomposition of the workspace is presented. In the horizontal direction, connection lines indicate a wholepart decomposition. It reveals constraints of, and relationships between, aircraft and airspace components, path control, locomotion physics, planning key functions, and the achievement of travel goals in terms of safety, production, and efficiency.

In this paper, the workspace content and boundaries are limited to trajectory planning functions in direct relation with conflict resolution and prevention during cruise flight and in situations with multiple aircraft. Functions related to aircraft control and stability, like staying within the flight envelope and accounting for passenger comfort, are kept out of the analysis. The time interval in which this workspace is analyzed is determined by the applicability of conflict management and is more or less situated between $60 \mathrm{~s}$ and around $15 \mathrm{~min}$. Below $60 \mathrm{~s}$, collision avoidance systems like the TCAS II must take over in order to prevent collision [17]. A 15-min upper threshold is chosen because the vast majority of conflict resolution and recovery maneuvers take less than $15 \mathrm{~min}$. In order to reduce problem complexity, only motion in the horizontal plane is considered in this paper. Extensions to the vertical plane, as well as combined vertical/horizontal representations, are currently being developed [2].

The hierarchy was developed using a top-down approach. At the highest abstraction level, i.e., the functional purpose, 
the ATP systems' main goals are identified as safe, productive, and efficient travel through airspace. In the context of conflict management, this means the efficient and productive prevention/resolution of conflict situations during cruise flight in the horizontal plane. At the abstract function level, the key functions describe how these goals can be achieved: while approaching the destination and limiting path deviation, spatial separation must be maintained at all times. Implicit coordination between different aircraft is beneficial for the realization of the key functions. On the general function level, the processes of aircraft locomotion and pilot control are described. Path control is done using the autopilot. Pilot control inputs relate to aircraft motion through aircraft kinematics and dynamics. On the bottom of the $\mathrm{AH}$, the physical form and functions are described using the airspace model, including the ownship and intruder aircraft. While these levels are relevant for the physical implementation of the system, they are not directly relevant to the work described herein. Thus, they are not further elaborated on.

\section{B. Workspace Key Functions}

In this section, the requirements for goal achievement are analyzed, and the relations with lower levels (locomotion, control, aircraft state, etc.) are identified.

1) Spatial Separation (Safety Goal): Spatial separation addresses separation from terrain and objects in space. Regarding separation from other aircraft, a separation standard is defined through a protected zone (PZ) centered around the aircraft [16]. Intrusion of this space is referred to as a "loss of separation," destroying the safety goal. In the horizontal plane, the PZ is a circle with a radius of $5 \mathrm{nmi}$ (Fig. 2). A conflict situation is defined as a future loss of separation within a given look-ahead horizon of $5 \mathrm{~min}$. Based on the ownship speed vector $\vec{V}_{\text {own }}$ and the intruder speed vector $\vec{V}_{\text {int }}$, a conflict detection algorithm can predict where the separation between both aircraft is minimal, the closest point of approach (CPA). In the situation shown in Fig. 2, the CPA lies inside the PZ, and separation will be lost within $5 \mathrm{~min}$ if no action is taken. Aircraft positions and PZ at the moment of CPA are also drawn in gray; these predictions are based on a new ownship speed vector $\vec{V}_{\text {new }}$. Clearly, separation can still be lost, even if the ownship turns away from the original PZ.

2) Destination Approximation (Production Goal): Production addresses certain "performance" to be produced by traveling. In general, this comes down to transporting persons or goods comfortably from A to B and deliver them on time. For trajectory planning, spatio-temporal deviation constraints, also known as time slots or time "gates," exist with respect to the destination, next waypoint or other point. For this paper, a simple requirement will be used, stating that the distance between aircraft and destination should decrease at all times. Thus, the pilot would nominally head straight toward the destination.

3) Path Deviation Minimization (Efficiency Goal): Efficient travel addresses economic fuel-efficient flight. Regarding conflict situations, the maximum spatial deviation from the path $\delta_{\max }$ is defined as the distance between the ownship's reference position and its actual position at CPA instance (see Fig. 3). The reference position is based on the prediction using the

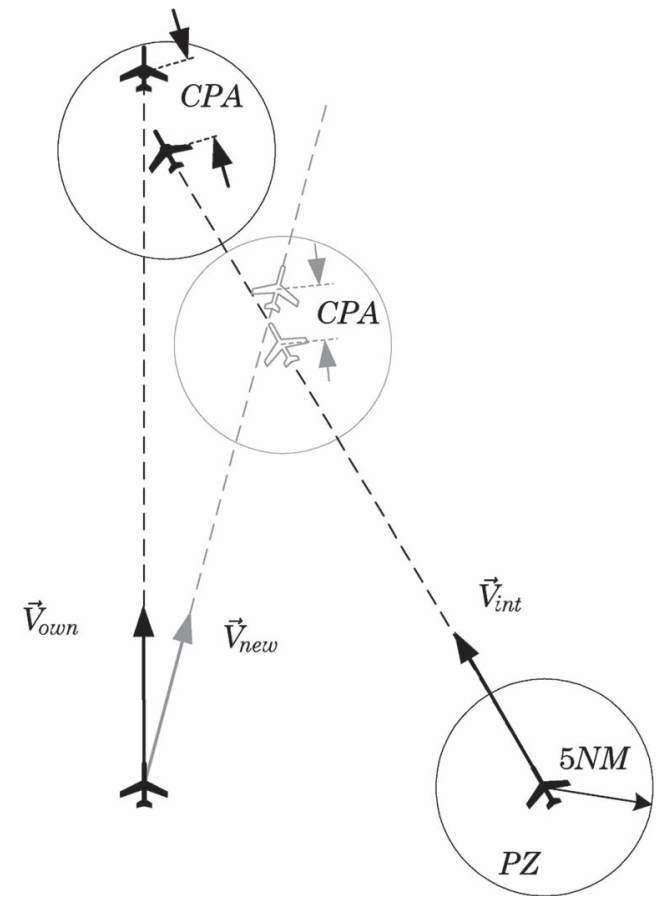

Fig. 2. Plan view of a conflict. The CPA is calculated for the current ownship speed vector (black) and a new owncraft speed vector (gray) after a turn.

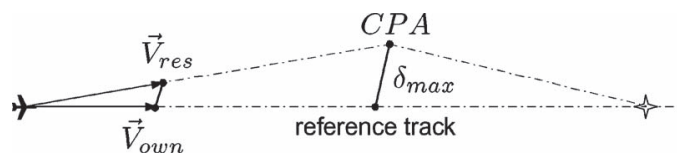

Fig. 3. Maximum path deviation $\delta_{\max }$ at instance of CPA.

original ownship's speed vector $\vec{V}_{\text {own }}$. The actual position is based on the resolution vector $\vec{V}_{\text {res }}$. After the CPA instance, the pilot will start a recovery maneuver to direct the aircraft back toward the original trajectory. The deviation due to conflict resolution is determined by two physical phenomena: the state change magnitude $|\Delta \vec{V}|$ and the duration of the resolution, the resolution time $t_{\mathrm{res}}$. The most efficient resolution minimizes the product of both factors

$$
\delta_{\max }=\left(|\Delta \vec{V}| * t_{\mathrm{res}}\right) .
$$

The state change magnitude $|\Delta \vec{V}|$ is obtained by subtraction of the original ownship's velocity vector $\vec{V}_{\text {own }}$ from the new ownship's resolution velocity vector $\vec{V}_{\text {res }}$

$$
\Delta \vec{V}=\vec{V}_{\text {res }}-\vec{V}_{\text {own }} .
$$

The deviation measure $\delta_{\max }$ is useful to compare different resolution maneuvers when considering their efficiency.

4) Implicit Coordination: Aircraft involved in the same conflict can have a mutual benefit from their maneuver actions if they are done in a coordinated fashion. For example, for 
two aircraft on a head-on collision course, coordination is fully exploited if both make a starboard turn maneuver. This way, the required resolution time or maneuver magnitude can be halved if compared to the case where only one aircraft maneuvers. As a result, the realization of other key functions becomes easier. Since no intent information is explicitly exchanged, a support tool should preferably "implicitly" assure coordination by the way it presents the conflict situation.

5) Goal Priority: Situations may occur in which not all goals can be met. In these cases, goal priority comes into play [22]. Safety is the highest priority, followed by production, then efficiency. For example, conflict situations may require trajectory deviations away from the destination.

\section{Functional Modeling of Aircraft Behavior}

The workspace analysis provides an overview of the content and structure of the planning workspace but does not yet provide us with any clues on how to create a meaningful display given that it does not answer the following questions.

By which aircraft (loco)motion can separation best be realized and how must it be visualized? How can we reveal the relation between a conflict resolution and path deviation, implicit coordination, and aircraft locomotion?

Thus, a step that facilitates the acquisition of workspace constraints in a way that abets visual presentation is necessary. A good interface design requires a clear presentation of the workspace constraints and support for goal-directed behavior to enhance quick (re)action upon behavior of the environment [21].

In the present context, the "system" dynamics are complex, as they depend on the behavior of multiple aircraft moving relative to each other. Traditional formulations of aircraft dynamics express aircraft (loco)motion through formulations given in the form of nonlinear state-space equations, e.g.,

$$
\begin{aligned}
& \underline{\mathrm{x}}=(p, q, r, \phi, \theta, \psi, u, v, w, x, y, z)^{\mathrm{T}} \\
& \underline{\dot{\mathrm{x}}}=f(\underline{\mathrm{x}}, \underline{\mathrm{u}}, t) .
\end{aligned}
$$

Although two (or more) of these equations (one for the ownship and one (or more) for any other nearby aircraft) describe the aircraft behavior adequately, a description of aircraft locomotion in this format is not useful for interface design as it fails to answer the two design requirements stated earlier.

First, the state-space formulation has too many degrees of freedom to be of practical use for goal-directed control. The input to the controlled system, the aircraft, is a function of time $\underline{\mathrm{u}}(t)$, and in principle, any input can be given (resulting in a high-dimensional "possible behavior" of the aircraft). Evaluating all possible inputs is impractical, and trying to display these options and their consequences is even more so. A lowdimensional description of aircraft behavior that uses inputs that match flight practice should be used. If not, the pilot will be unable to perform the described aircraft behavior.

Second, the state-space formulation is not related to any goaloriented behavior of the pilot. That is, for pilots, the main concern is not: "Which path will the aircraft follow if I do this?" but rather "Will it reach the destination without crashing into something?" Engineering descriptions for aircraft motion and motion paths, like the state-space description, do not directly relate to the goal-directed constraints in the planning workspace. Consequently, they are of little use in designing presentations that help pilots choose a trajectory.

In order to obtain goal-directed descriptions for aircraft behavior, or (loco)motion, one needs to describe the "function of" locomotion. Here, the word function is defined as "useful behavior" [11], i.e., behavior that is relevant to achieving one's ends. Functional modeling [13] describes the goal-relevant behavior of a system. By determining what types of possible behavior are functional to goal achievement, the possible alternatives for goal achievement are obtained.

Considering that we are matching the capabilities for action of a pilot-aircraft system, and their consequences as determined by its airspace environment (including other aircraft in the vicinity), parallels can be drawn with ecological psychology [7]. In this context, the affordances of the surrounding airspace describe the options available to aircraft and pilot, of which some are functional (avoiding other aircraft). A visualization of affordances of travel was already exemplified in the illustrations in Gibson's classic 1938 paper [8].

In the original EID framework applied to process control, the interface built for the power plant formed a new ecology/ environment for the operator. For travel, however, pilots already use an existing ecology in the form of the outside view and cockpit instruments, and therefore, natural ecological perception of affordances already exists. A pilot can, for example, predict a future collision by perceiving the angle between the ownship heading and the line extending to the intruder. If this angle remains constant in time, both travelers will eventually collide if no action is taken. A new interface tool should not substitute, but enhance this natural perception, by making visible those airspace affordances that are "hidden" from the naked eye [24]. In Fig. 2, one is not able to see by which maneuver the situation will or will not be resolved, even when the CPA and PZ are presented.

Different airspace elements yield different travel affordances. With respect to the ownship, intruder aircraft afford "collision" or "avoidance." With respect to the actual position of the aircraft, the destination or planned position affords "approximation" or "deviation." With respect to the aircraft traveling, air affords locomotion. With respect to the wings, air affords pressure difference. Note how airspace affordances relate to workspace constraints on different functional levels as well as decomposition levels of the AH (Fig. 1). The planning affordances that directly relate to more functional levels of abstraction are summarized in Table I. The next sections will investigate how the airspace environment should be presented, so that planning affordances can be perceived and fluently transformed into functional aircraft behavior, supporting the natural coupling between perception and action.

\section{Heading Travel Function}

The description of locomotion used to present the affordances must match the pilot flight practice and limit the number 
TABLE I

Planning Goals, Key Functions, AfFordances of Airspace Elements, and Maneuver Strategies for HTF AND SHTF. THE STRATEGY FOR IMPLICIT COORDINATION ONLY HOLDS FOR CONFLICT RESOLUTION

\begin{tabular}{|c||c||c||c||c|}
\hline planning goal & key function & affordance [airspace element] & HTF strategy & SHTF strategy \\
\hline safety & spatial separation & avoidance [other aircraft] & stay/get outside band & stay/get outside FBZ \\
\hline production & destination approximation & approach [destination] & keep destination in front & keep destination in front \\
\hline efficiency & deviation minimization & approach [original trajectory] & choose nearest band edge; & $\begin{array}{l}\text { limit state change } \\
\text { avoid FBZ origin }\end{array}$ \\
\hline safety/efficiency & implicit coordination & coordination [other aircraft] & choose nearest band edge & go to nearest FBZ leg \\
\hline
\end{tabular}

of degrees of freedom. This paper took the approach to select a reasonable locomotion model and to analyze if it was possible to present planning affordances. For cruise flight limited to the horizontal plane, the pilot controls the aircraft through heading and/or speed settings.

The first generation of locomotion models aimed at presenting planning affordances in terms of travel opportunities governed by manipulating only the aircraft heading [20]. The first model was based on constant speed and instantaneous heading changes. A second model, the heading travel function (HTF), included realistic turn dynamics. When considering these maneuver possibilities in a traditional plan view presentation where the ownship and other aircraft are shown, conflicts cannot easily be perceived. Knowing the velocities and trajectories of the involved aircraft conflicts can be detected, however, in a fast-time simulation, which is essentially the basis for current conflict detection and avoidance systems.

If the PZ is shown at the moment of CPA, as in Fig. 2, one can see whether separation is lost for the current trajectory prediction based on vector $\vec{V}_{\text {own }}$. The CPA, however, is a characteristic of 4-D space; it only remains constant when the aircraft locomotion states are not changed. If a turn maneuver to starboard is made, the predictions for the new vector $\vec{V}_{\text {new }}$ show that the CPA has moved and that the conflict is not resolved at all. Thus, presenting the CPA does not enable pilots to "see" which maneuvers would resolve the conflict. Thus, in plan view orthogonal space, maneuvers cannot be directly linked to separation. It is unclear which paths will eventually stay out of or enter the intruder aircraft PZ.

Conflicts can, however, easily be seen in a relative velocity field. In an intruder-centered reference frame, the motion of the ownship is expressed relative to the intruder, and the intruder's PZ is pinpointed in space. In this field, aircraft maneuvers can be linked to spatial separation. Subtracting the speed of the intruder yields a representation that shows travel of the ownship in a relative space, i.e., the space and travel relative to the intruder aircraft. On the left-hand side of Fig. 4, the HTF expresses which of the possible ownship paths will stay out of the intruder aircraft PZ. Note that the effects of turn maneuver dynamics are included, as can be noticed by the bended shape of each ownship path, together forming the set of relative motion paths that will realize separation.

\section{A. Heading Bands Presentation}

1) Safety: Travel in "relative space" is not a practical way to present travel options to the pilot. It is preferred to directly

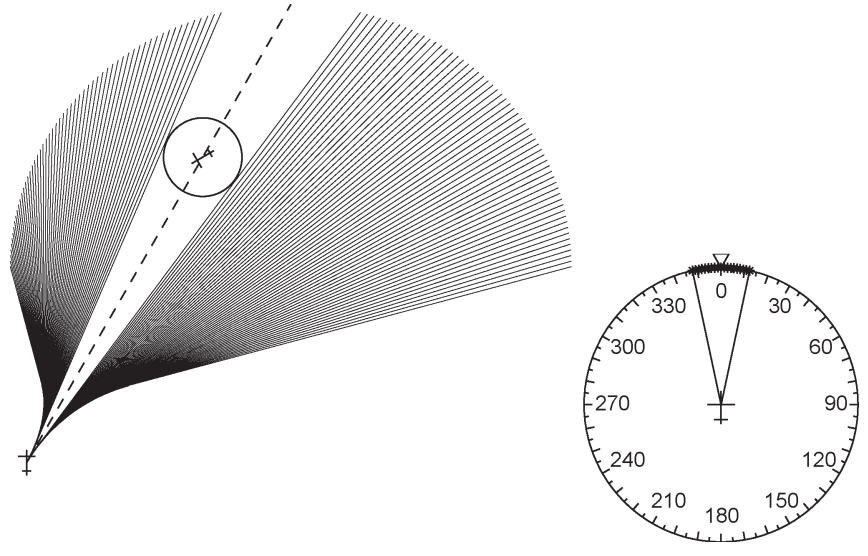

Fig. 4. (Left) HTF in intruder-centered reference frame. (Right) The heading band shows turns that will lose separation.

present which turn maneuvers resolve the conflict(s) and which do not. This way, the so-called heading bands can be created. ${ }^{3}$ That is, the interval of turns that will lead to loss of separation is presented on the heading scale through colored heading bands (see the right-hand side of Fig. 4).

Since the turn geometry is included in the functional locomotion model, as soon as a turn to a conflict-free heading is initiated, the heading band edges remain stable, i.e., they maintain the same heading values. Note that our first locomotion model, based on instantaneous heading changes, did not take the turn dynamics into account and therefore failed to accurately predict the turn maneuver needed to get out of the heading band. From the heading band presentation, a simple turn strategy can be used to resolve a conflict, i.e., "turn out of the heading band" (see Table I).

2) Production: The production goal accomplished by the strategy to "head toward the destination" is easily realized by limiting possible heading changes for conflict resolution to $90^{\circ}$. Generally speaking, for trajectory planning, pilots can always turn "toward" the destination: making a turn so that the destination lies in the extension of the current heading.

\section{B. Hypothesis for Efficiency and Implicit Coordination}

In order to set up a "turn" travel strategy that assures cooperation between two aircraft, the following planning rule

${ }^{3}$ It is important to emphasize the distinction between a "travel function," such as the HTF, and an "interface concept," such as the heading bands. A travel function is a formulation of aircraft motion presenting a set of travel options to the pilot, whereas an interface concept is a presentation format that visualizes workspace constraints/affordances in terms of these travel options. 
was defined: "A conflict must be resolved by taking a turn toward the closest heading band edge." Because of the symmetrical conflict geometry, this will generally result in cooperative maneuvers. Both aircraft can simultaneously initiate these maneuvers based on the position of their heading marker inside the heading band. Furthermore, an efficiency hypothesis can be explored: A conflict resolution maneuver toward the nearest band edge results in the smallest lateral deviation $\delta_{\max }$. A complete overview of the HTF planning strategies with respect to the key functions is presented in Table I.

Research showed that the angular proximity of the heading band edge is not proportional to the maximum lateral deviation [18]. The perception of both heading band edges and then the strategy of steering toward the closest edge does not necessarily result in the smallest $\delta_{\max }$ of the two possible turn resolutions. Some trajectories to resolve a potential conflict bring the aircraft on a parallel course. If the speed vectors of both aircraft are approximately equal, resolution time $t_{\text {res }}$ becomes very high. As a consequence, a very large path deviation is obtained. Although steering toward the nearest heading band edge results in the smallest state change $\Delta \vec{V}$, the bands provide no measure of the duration of a conflict resolution. In some situations, a small $\Delta \vec{V}$ is accompanied by an extremely large $t_{\text {res }}$, causing the product of both terms, the path deviation $\delta_{\max }$, to become very large as well.

Another aspect of the HTF travel strategy is that it does not consider aircraft speed changes. In previous research on P-ASAS, the heading bands' width and position showed to be very dependent on speed [9]. When speed changes, heading bands move, split up, shrink, or expand, changing the range of conflict-free headings. The unsteady heading band behavior based on the current speed caused P-ASAS to be extended with a multiple heading band presentation, based on a set of different speeds around the current speed [9].

Clearly, the presentations based on the HTF only present separation in an invariable way as long as the pilot avoids changing speed. However, satisfying other goals might require these speed changes to, for instance, compensate for time deviation along track. In that case, the bands lose their current width and position, and it becomes useless and even misleading to show separation by heading bands. It is also doubtful whether implicit cooperation between aircraft can be perceived when multiple conflicts occur simultaneously. In that case, multiple heading bands belonging to different aircraft will appear and overlap each other on the heading scale.

An alternative for visualizing separation on a traditional plan view display is the presentation of "forbidden areas" where current trajectory prediction (conflict zone) and potential trajectories (no-go zone) will lose separation [5]. However, since these are also based on heading changes, they have the same deficiencies as the heading bands. That is, although adequate for pure heading changes (at constant speed), the shape is dependent on speed and sometimes fails to visualize more efficient conflict resolutions. Similarly, speed bands based on locomotion models that use constant heading have the same drawbacks as the heading bands: They are sensitive to heading changes, and they fail to present efficient conflict resolutions. Presentations that use both speed and heading bands simultane- ously do not improve much either as changing the state within one band changes the appearance of the other band. Clearly, a fundamentally different approach is needed.

\section{Speed-Heading Travel Function}

The interaction between speed and heading must be fully understood. Hence, in the third locomotion model, the speedheading travel function (SHTF) model, the aircraft behavior was modeled by combined speed and heading change maneuvers [18]. At this stage, the aircraft dynamics were neglected, however, due to the increased complexity of expressing combined heading and speed changes. The main challenge lies in finding an invariable visualization for efficient conflict resolution in such a way that it supports a travel strategy that yields implicit coordination between two or more aircraft.

\section{A. Forbidden Beam Zone and State Vector Envelope}

1) Safety: As mentioned in the previous section, (loco)motion can be better related to spatial separation constraints in a relative velocity plane. Within this plane, a beam-shaped area can be defined, as shown in Fig. 5(a), by two lines originating from the own position and tangent to, respectively, the left and the right side of the $\mathrm{PZ}$ of the intruder. This zone is referred to as the forbidden beam zone (FBZ). In this example, the relative velocity vector $\vec{V}_{\text {relown }}$ is inside this area, and therefore, the aircraft will eventually enter the PZ, and separation will be lost. The minimum separation distance at CPA instance is indicated by $d$.

Separation can be realized by actions that cause the relative velocity vector to lie outside the FBZ. The nearest "exit" point on the FBZ is indicated by circled number 1 in Fig. 5(a). If $\vec{V}_{\text {rel }}$ iwn is moved to this position, the resulting resolution position of the CPA is then indicated by $\mathrm{CPA}_{\text {res }}$. This particular resolution is identical to the resolution given by the "voltage potential" method used in P-ASAS [9]. Since the relative vector is constructed by the own speed vector $\vec{V}_{\text {own }}$ and the intruder vector $\vec{V}_{\text {int }}$, spatial separation can be realized by a vector state change of the own speed vector, the intruder speed vector, or a combination of both.

In an ownship-centered speed-heading vector space shown in Fig. 5(b), the FBZ remains visible with respect to the own speed vector which is centered and placed upward. The geometrical relations in Fig. 5(a) remain identical, but the space is now pinpointed around the origin of the ownship speed vector $\vec{V}_{\text {own }}$. In the same space, one can draw an arc of constant speed inside the FBZ, revealing one (or more) heading bands. In Fig. 5(c), the band is shown for the current speed (including arrow heads) and three other speeds (one band for slower speed and two bands for higher speeds). It shows why the position and shape of the heading bands in the HTF are sensitive to speed changes and how this relates to the shape of the FBZ. Similarly, speed bands are sensitive to heading changes, and they also fit inside the FBZ. This explains how the state bands based on a single variable (like heading in case of the heading band) behave when other control variables (in this case, the speed) are manipulated. In [9], this behavior was reported in the P-ASAS state bands 


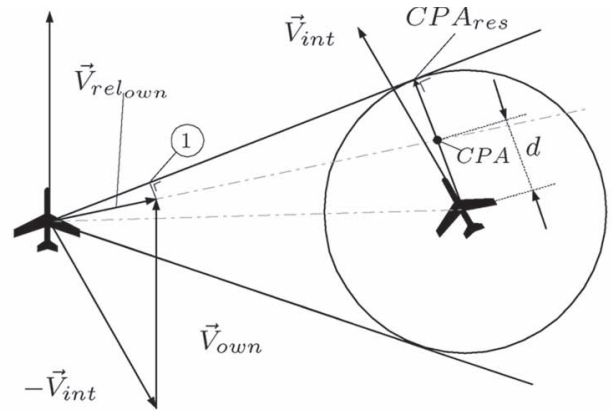

(a)

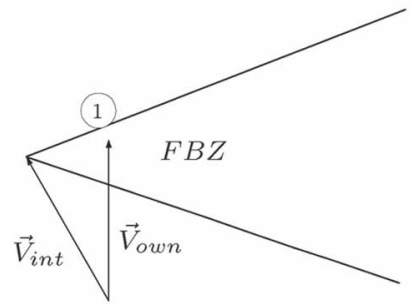

(b)

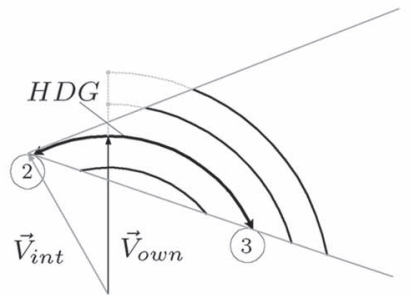

(c)

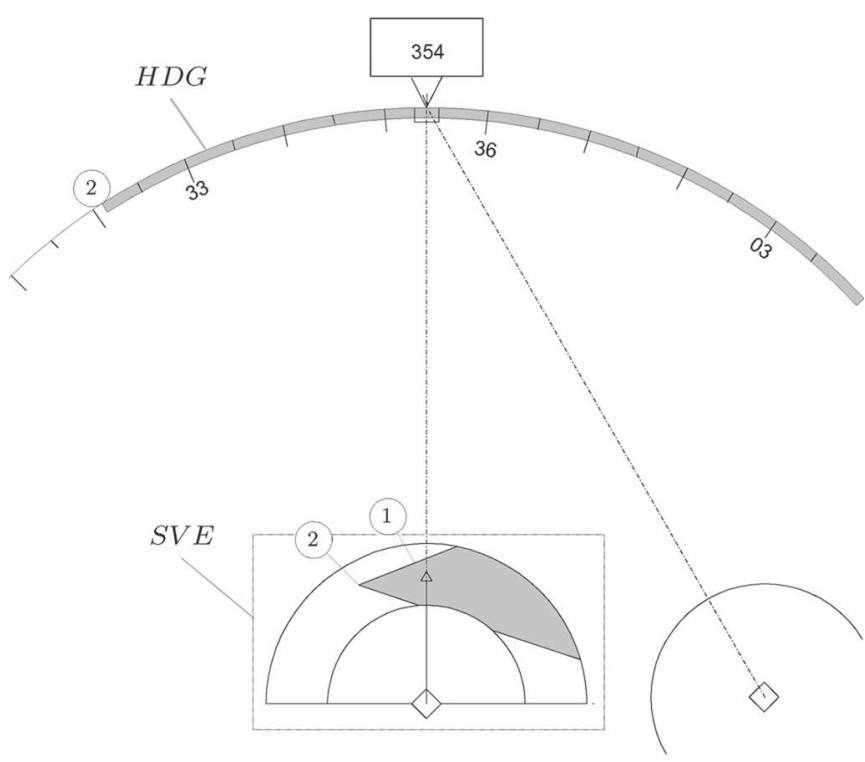

(d)

Fig. 5. Presentation of a conflict situation. Numbers 1 to 3 present possible conflict resolution maneuvers based on FBZ (1) and heading band HDG $(2,3)$. $\mathrm{CPA}_{\text {res }}$ is the resolution position of the CPA. (a) Relative motion of ownship with respect to intruder in an intruder-fixed reference frame. (b) FBZ in an ownship-centered speed-heading space. (c) Heading band (HDG) for different speeds in an ownship-centered speed-heading space. (d) SVE and heading band on an ND.

designed for preventing the triggering of new conflicts during conflict resolution.

2) Efficiency: On the FBZ in Fig. 5(b), the resolution maneuver that gives the smallest state change $|\Delta \vec{V}|$ is marked with the circled number 1 . This maneuver is identical to the resolution proposed in the previous paragraph. The resolution time $t_{\text {res }}$ equals the distance between both aircraft, $\Delta \vec{X}$, divided by the relative approaching velocity of the resolution speed vector $\left|\vec{V}_{\text {res }_{\text {rel }}}\right|$

$$
t_{\mathrm{res}}=|\Delta \vec{X}| / \vec{V}_{\mathrm{res}_{\mathrm{rel}}} .
$$

Since $\Delta \vec{X}$ is constant at the instance of choosing a resolution, $t_{\text {res }}$ is inversely proportional to $\left|\vec{V}_{\text {res }_{\text {rel }}}\right| \cdot\left|\vec{V}_{\text {res }_{\text {rel }}}\right|$ is the difference between the resolution vector $\vec{V}_{\text {res }}$ and the vector pointing to the origin of the $\mathrm{FBZ}, \overrightarrow{\mathrm{FBZ}}$ origin

$$
t_{\text {res }}=c /\left|\vec{V}_{\text {res }}-\overrightarrow{F B Z}_{\text {origin }}\right|
$$

where $c$ is a constant. $\overrightarrow{F B Z}$ origin is equal in magnitude and direction to the intruder speed vector $\vec{V}_{\text {int }}$. As a consequence, $t_{\text {res }}$ can be perceived by the distance between the resolution velocity vector $\vec{V}_{\text {res }}$ and the intruder vector $\vec{V}_{\text {int }}$

$$
t_{\mathrm{res}}=c /\left|\vec{V}_{\mathrm{res}}-\vec{V}_{\mathrm{int}}\right|
$$

where $c$ is a constant. If the resolution vector $\vec{V}_{\text {res }}$ lies far away from the intruder vector $\vec{V}_{\text {int }}$, the resolution time $t_{\text {res }}$ will be small. When $\vec{V}_{\text {res }}$ has nearly the same heading and speed as $\vec{V}_{\text {int }}$, then the resolution time will go to infinity as nearly "parallel" trajectories are flown.

The description for the maximal deviation $\delta_{\max }$ in (1) can now be replaced by

$$
\delta_{\max }=c *\left(\left|\vec{V}_{\text {res }}-\vec{V}_{\text {ref }}\right| /\left|\vec{V}_{\text {res }}-\vec{V}_{\text {int }}\right|\right)
$$

where $c$ is a constant. $\delta_{\max }$ is proportional to the quotient of resolution state change magnitude $\left|\vec{V}_{\text {res }}-\vec{V}_{\text {ref }}\right|$ and the distance between resolution state and FBZ origin $\left|\vec{V}_{\text {res }}-\vec{V}_{\text {int }}\right|$. Since an efficient conflict resolution comes down to minimizing the maximal deviation, an efficient conflict-free travel strategy will require a small state change away from the FBZ origin.

If one now considers the heading band for the current speed in Fig. 5(c), two resolution maneuvers are possible: a port turn to the point indicated by circled number 2 or a starboard turn to point 3 . It can be easily seen that the state change magnitude $|\Delta \vec{V}|$ of resolution maneuver 2 is smaller than that for solution 3 . The heading band visualizes this magnitude, and the HTF travel strategy tried to use this measure to form a travel strategy for cooperative efficient conflict resolution. However, since solution 2 , the preferred resolution for the strategy, lies very close to the intruder vector $\vec{V}_{\mathrm{int}}$, the resolution time $t_{\mathrm{res}}$ will be very large and the maximal deviation $\delta_{\max }$ will be several times larger than that for resolution maneuver 3 . The FBZ visualizes both the state change magnitude $|\Delta \vec{V}|$ and the resolution time $t_{\text {res }}$ so that deviation can be more effectively minimized. The resolution maneuver toward the point indicated by number 1 in Fig. 5(b) is the best resolution option. Again, note that this is identical to the resolution provided by P-ASAS [9].

3) Production and Other Constraints: The presentation of the FBZ can be further adapted by introducing other workspace constraints/affordances. Limitations to aircraft performance (constraint at physical level), such as maximum and minimum values for aircraft velocity, can be applied. Due to productivity (a more functional workspace constraint), the heading change is limited to $90^{\circ}$ port and starboard in order to show travel opportunities that will decrease the distance between the aircraft and the destination. The resulting presentation is called the SVE 

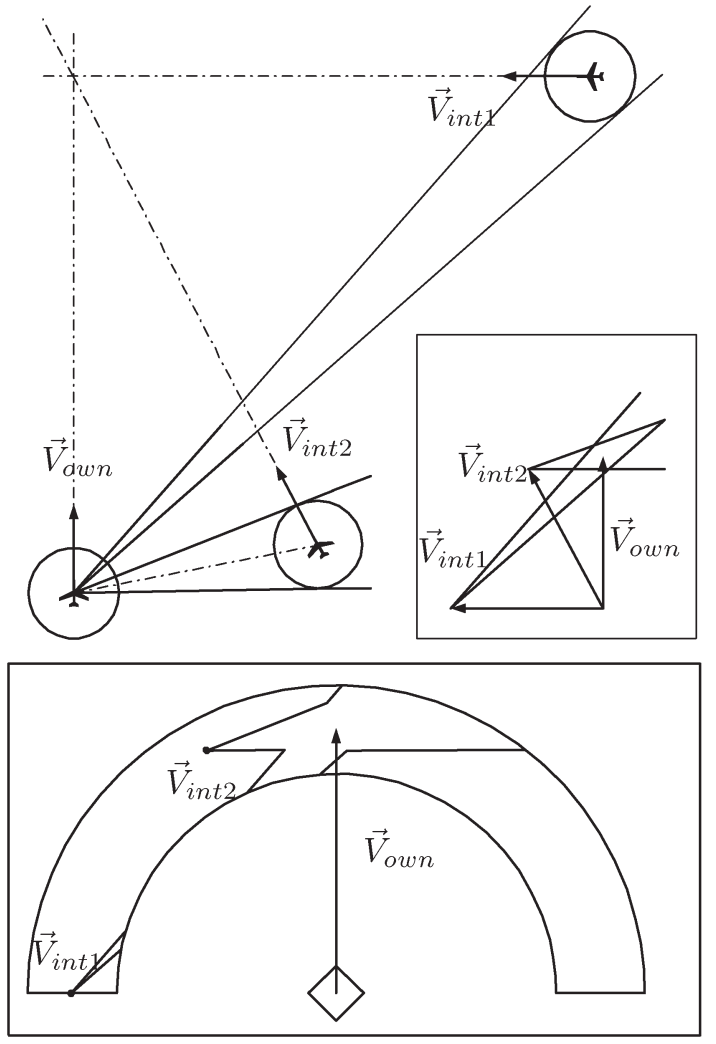

Fig. 6. Combination of two FBZs due to conflict with two intruders.

and will be used as the interface concept related to the SHTF travel function. Fig. 5(d) shows the SVE at the bottom of the navigation display (ND). Note that the SVE contains the FBZ and that its boundaries are imposed by aircraft performance and productivity constraints.

\section{B. Multiple Conflicts and Implicit Coordination}

1) Multiple Conflicts: When multiple conflicts occur simultaneously, several FBZs can be shown superimposed on each other. Fig. 6 shows multiple conflicts with two intruder aircraft. $\vec{V}_{\text {own }}$ is the speed vector of the ownship; $\vec{V}_{\text {int1 }}$ and $\vec{V}_{\text {int2 }}$ are the speed vectors of the two intruders. The SVE is shown at the bottom of the figure. The geometric relation between the beam position and orientation on the one hand, and intruder position and speed vector on the other hand, allows pilots to correlate FBZs with aircraft symbols on the ND. The FBZ origin position represents the intruder speed vector. The direction of the opening of the beam reveals the intruder relative position.

2) Implicit Coordination: In case of a conflict, the geometry of the FBZ from the perspective of one aircraft is complementary to the perspective of the other aircraft. In Fig. 7, one can see that because of the symmetry, the closer one's aircraft vector endpoint is located to one leg of the FBZ, the closer the other aircraft's vector endpoint will be to the opposite leg. Hence, the resolution of aircraft 1 (labeled with number 1) is complementary to the resolution of aircraft 2 (labeled number 2). Therefore, the motion of the FBZ due to the maneuver of aircraft 1 is in the opposite direction of the motion induced

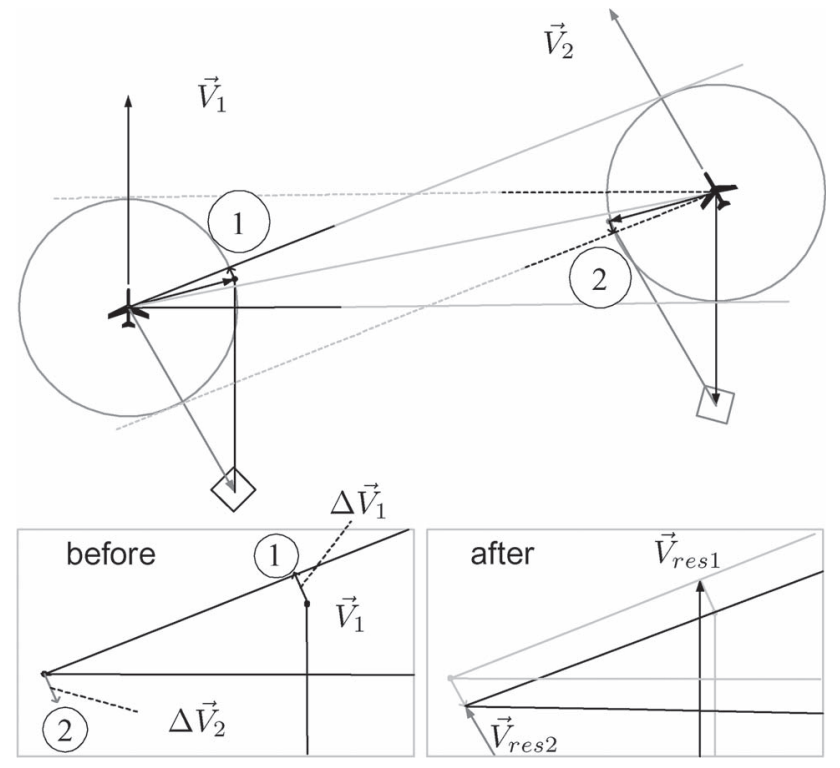

Fig. 7. Implicit coordination between two aircraft.

by the maneuver of aircraft 2 . On the bottom of Fig. 7, one can see two close-ups on the FBZ from the perspective of aircraft 1 before (left) and after (right) the resolution maneuvers are done. It illustrates the situation before (gray) and after (black) maneuvering. Because both aircraft maneuver, the magnitude of the resolution maneuver could be half of the indicated resolutions. In this way, each pilot can move to the FBZ leg that is situated closest, yielding simultaneous cooperative maneuvers, without the need to exchange intent information.

\section{Spatio-Temporal Forbidden Beam Zone Dynamics}

The changing relative aircraft positions cause the FBZ to expand. Both ownship and intruder maneuvers result in translation and/or rotation of the FBZ. A proper analysis of the FBZ dynamics is needed to understand how these dynamics affect pilot decision making and conflict awareness.

1) FBZ Expansion: In Fig. 8(a), the ownship speed vector $\vec{V}_{\text {own }}$ at time $t_{0}$ lies inside the FBZ, and during the time that the ownship is approaching the intruder aircraft and, therefore, the PZ, the FBZ-beam width will expand. The different effects of the maneuver and the expansion are indicated with man and exp, respectively. The FBZ is drawn at the beginning $\left(t_{0}\right)$ and at the end $\left(t_{1}\right)$ of the maneuver in an intruder-fixed reference frame [Fig. 8(a)], an intruder-fixed speed-heading space [Fig. 8(b)], and an ownship-fixed speed-heading space as shown on an SVE [Fig. 8(c)].

The closer the conflict comes to the actual loss of separation, the more significant the expansion rate will be, resulting in a "sweep movement" at the end, as shown in Fig. 9. The FBZ is drawn for the conflict situation at current time $t_{0}$ and four instances in the near future ( $t_{1}$ to $t_{4}$ using equal time steps) when both aircraft do not maneuver. Since the ownship's vector is located exactly on the upper FBZ leg, this leg will not sweep at all, at least not until the CPA point is passed. The perception of an increased expansion rate gives the pilot an indication 


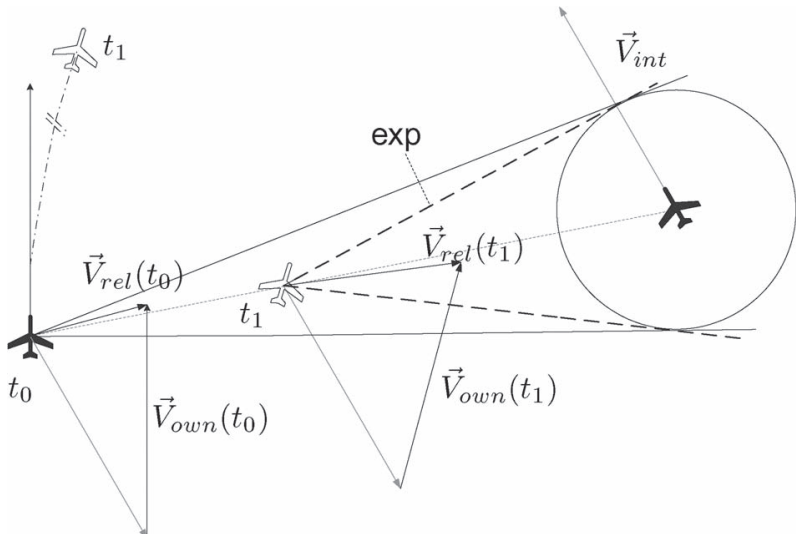

(a)

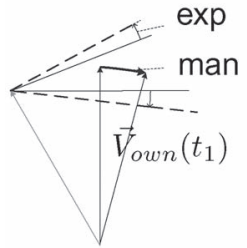

(b)

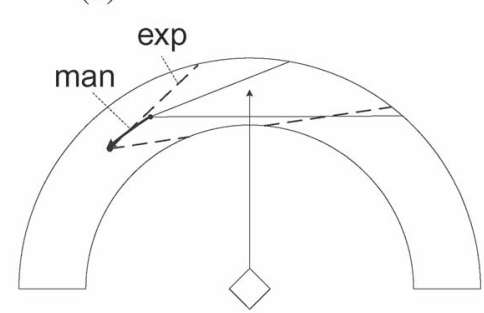

(c)
Fig. 8. Expansion of the FBZ (top) when doing nothing or (bottom) when executing a resolution maneuver. (a) Relative motion of ownship in an intruderfixed reference frame. (b) Maneuver in intruder-fixed speed-heading space. (c) Maneuver in SVE (ownship-fixed speed-heading space).

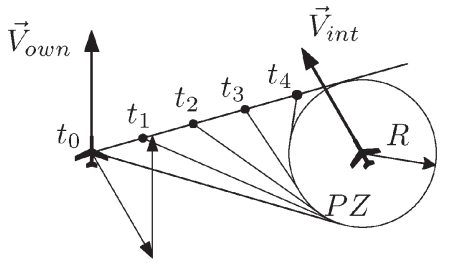

(a)

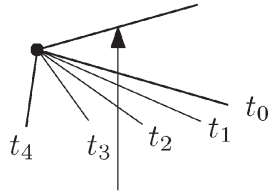

(b)
Fig. 9. FBZ expansion and sweep movement. (a) Relative motion of ownship. (b) Ownship-fixed speed-heading space.

of conflict urgency. A pilot could estimate whether it is still possible to go out of the FBZ or trespass it. Note, however, that it does not guarantee that separation is indeed feasible, unless exact aircraft maneuver dynamics are used in the model. Furthermore, the expansion rate only becomes significant when time-to-CPA is very low (less than $1 \mathrm{~min}$ ), and by that time, a collision avoidance system is employed.

2) Intruder Maneuvers: Intruder maneuvers can also be directly perceived by translation of the corresponding FBZ. Due to the displacement of the intruder velocity vector $\overrightarrow{\vec{V}_{\text {int }}}$, "fixed" to the origin of the FBZ, the entire FBZ is translated. When the intruder is inside the FBZ, a cooperative maneuver is intuitively realized by steering in the opposite direction of the intruder maneuver, resulting in a lower conflict resolution time or a larger separation. Similarly, when the intruder aircraft is outside the FBZ but makes a "hostile maneuver," i.e., toward an FBZ leg, the pilot can now prevent intrusion by initiating exactly the same maneuver as the intruder.

\section{Hypothesis for a Travel Strategy With the SVE}

Considering the aforementioned analysis, the following travel strategy will efficiently resolve and prevent a conflict situation with an intruder aircraft.

1) Safety: Stay out and get out of the FBZ before the aircraft enters the PZ of other aircraft.

2) Production: Keep or get the destination in front.

3) Efficiency: Minimize the deviation of the original trajectory path by optimizing two factors.

a) Limit the heading and speed deviation for the resolution/prevention maneuver: "For resolution (when intruder is not maneuvering), go to the closest resolution state on the side of the closest FBZ leg," and "for resolution and prevention (when intruder is maneuvering), move against and along the direction of the FBZ translation, respectively."

b) Limit the duration of the conflict resolution/prevention time: "Stay away from FBZ origin points."

Through the use of the efficiency travel strategy, implicit coordination of a conflict between two aircraft is guaranteed as long as the optimal resolution (or, at least, a resolution that lies on the side of the shortest FBZ-leg) is available. Although more complicated, this strategy can also be applied to multiple conflict situations. As long as the pilot takes a resolution that is situated on the side of the shortest FBZ leg of each individual pair of FBZ legs (one pair for each conflict), implicit coordination between each individual conflict is achieved.

Situations may occur, however, where the resolution space at the side of the FBZ is unavailable due to other mapped constraints. In such a situation, the pilot would have no other option but to perform a counteractive maneuver to the furthest FBZ leg. Given the assumption that no intent information is exchanged, the intruder cannot be informed about this maneuver. The addressed problem is inherent to the travel strategy. Therefore, an additional rule is needed: if the closest FBZ leg is not available, the ownship should not maneuver at all. The intruder aircraft resolves the conflict alone.

\section{E. Presenting the State Vector Envelope on the Navigation Display}

The SHTF expresses possibilities for motion in terms of instantaneous speed-heading state changes. In the intruder-fixed reference frame, the FBZ visualizes how both the ownship and intruder speed vector afford efficient and cooperative conflict resolution and prevention. The FBZ geometry remains identical when translated to an ownship-centered speed-heading state space [see, e.g., Fig. 5(b)]. Such presentation formats were also proposed for the design of maritime collision avoidance systems [4], [10], [12].

The SVE interface is overlaid on the ND at the bottom [Fig. 5(d)]. The main disadvantage of such an overlay is the risk of confusion between information on the plan view of the ND, a 2-D ownship-fixed spatial field, and the speed-heading space, a vector field. Still, the advantages seem to cancel out this disadvantage.

First, mapping the SVE on the ownship position of the ND relates the aircraft in the spatial space (the ND) with the FBZ in 
the speed-heading space. The pilot can observe speed, heading, and relative position of aircraft in the vicinity and also how these intruder characteristics affect the ownship's travel options. The use of speed-heading space (motion) and plan view (airspace) for presenting constraints therefore enhances the natural ecology that pilots have and use when moving through airspace. Second, possible maneuvers in the speed-heading space can be translated to the ND by (mentally) plotting a course in the short/middle term (planning). Third, the mapping further enables the presentation of workspace constraints in the spatial plane, the speed-heading space, or both. For separation, the intruder aircraft symbol in the spatial plane shows how much the actual separation is, whereas in the speed-heading plane, the FBZ shows if separation will be lost in the future and by which actions pilots can efficiently resolve the situation. The interface designer now has two possible "spaces" in which to present constraints, paving the way for more extended or integrated support tools.

An alternative to mapping the envelope would be to decompose the speed-heading space into separate speed and heading bands, but then, a part of the conflict information contained by the FBZ position and shape would be hidden: combined speedheading maneuvers, intruder behavior, and multiple conflicts.

\section{ECOlogical Interface Design Properties OF THE INTERFACE}

EID addresses how information should be presented and how to support operators to deal with novelty and change. The skills, rules, and knowledge (SRK) taxonomy of Rasmussen is a framework for describing the mechanisms that people have for processing information [14]. It defines three levels of cognitive control when describing human behavior in reaction to available information. EID aims to support all three levels of cognitive control, while not forcing the operator to control at a higher level than necessary, saving cognitive resources [23]. In this section, it will be discussed how the SVE interface supports these EID principles.

The SVE interface supports skill-based behavior by enabling the pilot to act on directly perceivable constraints. The speedheading state vector should be kept inside the envelope and outside the FBZs. Through the path control (speed and heading settings on the autopilot), the pilot can directly manipulate the goal state.

Rule-based behavior involves associating familiar perceptual cues in the world with an action or intent. There should be a consistent one-to-one mapping between the workspace constraints and the perceptual information on the SVE interface. Domain constraints related to separation and path deviation are mapped into perceptual cues: the distances between FBZ origin and state vector, between FBZ legs and state vector, and the movement of the FBZ give input to the pilot's travel strategy for efficient conflict resolution and prevention. There is a oneto-one mapping between the type of conflict situation and the FBZ presented on the SVE interface. Over time, different avoidance strategies can be tested, selected, or discarded depending on their efficiency. Heading constraints (production goal) and speed constraints (aircraft performance) mark the capabilities of the locomotion function and are mapped on the interface (boundaries of the SVE).

Knowledge-based behavior involves analytical problem solving based on a symbolic mental model. The interface should present the content and relations identified by the $\mathrm{AH}$ model of the workspace. The separation function on the abstract function level of the AH is revealed by the FBZ, as explained earlier. The relation between the generalized functions (locomotion, path control) and the abstract functions lies in the formulation of separation in terms of aircraft (loco)motion. The locomotion prediction depends on the possible speed and heading settings given by the pilot (path control function). The presentation of the "functional information" through the FBZ is built up out of physical information of airspace elements (physical function level): The intruder's position is revealed by the FBZ orientation pointing toward the symbol on the ND. Because of this relation, each aircraft on the ND can be related to an FBZ on the SVE, also in case of multiple simultaneous conflicts. The position of the ownship speed vector relative to the FBZ also reveals how it will pass the intruder (front, back, left, right, or a combination).

Intruder velocity is shown by the FBZ origin and can be directly compared to the ownship's velocity vector. Intruder maneuvers (vector changes) are visible by the movement of the FBZ. The effects of the intruder behavior on the constraints are clearly visible and allow the pilot to react properly, even if the behavior is unexpected and unanticipated for. Finally, the expansion rate of the FBZ is a measure for the time-to-CPA, i.e., a measure of "conflict urgency." The higher the expansion rate, the more critical it becomes to decide for and start a resolution maneuver.

\section{Pilot Evaluation}

In a fixed-base part-task flight simulator, the SVE interface has been evaluated by a brief test experiment with six professional civil airline pilots.

The purpose of the evaluation was to verify the safety and efficiency of conflict resolutions and to obtain a first impression of pilot acceptance and situation awareness. It is stressed that the evaluation was not aimed at covering all our claims made earlier. For example, it did not consider implicit coordination. Rather, scenarios included hostile intruder behavior to test the robustness of our interface against unanticipated behavior.

\section{A. Procedure and Setup}

A set of five conflict scenarios with two intruder aircraft was simulated. Each scenario had a specific conflict geometry. Pilots were asked to fly a track between two waypoints in cruise conditions. At a given moment, a conflict situation occurred with two intruder aircraft. Pilots were instructed to conduct a maneuver that would result in a safe and efficient conflict resolution using the speed-heading maneuver strategy. When the intruder aircraft had passed by, pilots were told to start a recovery maneuver, i.e., going back to the original cruise speed and head toward the next waypoint in order to continue flight on the original trajectory. 


\section{B. Description of the Simulation}

A Boeing 747-200 aircraft was simulated, flying at $30000 \mathrm{ft}$. Initial velocity was $0.8 \mathrm{Mach}$, approximately $240-\mathrm{m} / \mathrm{s}$ ground speed. The autopilot was enabled; speed and heading could be set on a simulated mode control panel using a mouse.

Given the actual speed, the "conflict detection" algorithm detected a future spatial separation violation (5-nmi reference) within a 5-min look-ahead time. The "FBZ drawing" algorithm used a look-ahead time of $15 \mathrm{~min}$ in order to show less urgent conflicts inside the SVE map when a resolution strategy was chosen for the actual conflict.

Each intruder was simulated with a propagation model that defined an initial trajectory by its position, ground speed, and heading. At a given time, a resolution maneuver with a different ground speed and heading was triggered. When the intruders passed each other, they headed back to their original trajectory path. The resolutions were pilot-like and caused a spatial separation between 5 and $10 \mathrm{nmi}$. The maneuver dynamics included a simple turn geometry and a constant longitudinal acceleration. Both intruder aircraft only resolved the conflict with each other and neglected the conflict situation with the owncraft. It was therefore possible for the intruders to make counterintuitive or even hostile maneuvers at the time they initiated the resolution or recovery maneuver. Pilots were informed about this intruder behavior during the briefing.

Three "normal" scenarios were designed in such a way that using the travel strategy at detection time would lead to the most efficient resolution. In the two other scenarios, the intruder behavior during resolution, i.e., before passing the CPA point, changed the desired resolution strategy.

\section{Results}

Safety was measured by loss of separation and the minimum separation distance, and efficiency by the maximum path deviation. In the normal scenarios, out of 18 runs, 14 times pilots applied the most efficient resolution strategy, and 1 time a suboptimal one. The three less-efficient strategies resulted in a path deviation that was at least twice as high as necessary. In one of the scenarios, a hostile recovery maneuver near CPA instance caused a minor loss of separation of a few hundred feet.

In case the unexpected intruder behavior changed the optimal resolution maneuver strategy for the own aircraft, pilots would have to cross the forbidden area. However, our pilots did not feel confident enough to do this. In one scenario, two equally efficient resolution strategies existed, a port and a starboard maneuver. Four pilots decided to do a starboard turn maneuver. Due to the resolution maneuvers of the intruders, this resolution would lead to a path deviation $\delta_{\max }$ three times higher than for the port maneuver. One out of four pilots decided to replan the strategy and cross the FBZ to take the more efficient option. In another scenario, the optimal solution was a small triangular area on the SVE that would disappear just before passing each other due to a hostile intruder recovery maneuver. Four pilots avoided this strategy from the start, taking a less efficient but more safe resolution strategy. One of the two pilots who did chose the optimal strategy eventually lost separation passing by at $2.3 \mathrm{nmi}$.

\section{Pilot Comments and Pilot Acceptance}

Pilot acceptance and conflict awareness were evaluated through the personal feedback of pilots and a questionnaire. All pilots indicated that the SVE interface was useful, but more practice and experience would be needed for a better comprehension and use of it. All indicated that they were able to correlate envelope lines to particular intruder aircraft and also reason about how aircraft will pass each other (back, front, left, or right), but they again mentioned that more practice was needed. Pilots recommended to keep the beam shape of each individual FBZ visible, i.e., draw the entire beam until the maximal velocity limit, including the part below the minimal velocity, and staple different FBZ on each other without merging the lines. The acceptability of, and confidence in, trespassing the FBZ area varied among pilots.

The FBZ expansion was easily perceived. All pilots acknowledged that the evolution of the envelope form was noticed better when the intruder aircraft came closer to the owncraft. However, it was difficult to predict the expansion rate of the FBZ. The lack of awareness about the future position of the beam edges made it difficult to exactly determine the right maneuver needed to get out of the FBZ.

All pilots commented to have a reasonable notion on conflict urgency. Three pilots indicated that they used the FBZ size to build up this notion. All pilots used spatial proximity of the intruder aircraft on the ND. Two pilots also used relative approaching velocity of the aircraft symbols on the ND. It was not clear to them how much time was left before collision or, alternatively, how much time was left to start a resolution maneuver. Pilots indicated that they particularly wanted to perceive the relative velocity of the intruder aircraft.

During the experiment, most pilots did not explicitly state that the intruder aircraft were maneuvering. They spoke about movements of the envelope lines and how to react upon them. When a hostile intruder maneuver was done just before passing by, however, pilots clearly identified the maneuver.

\section{Discussion}

A work domain analysis was made of ATP, identifying behavior-shaping workspace constraints, related to key functions like separation and path deviation. These were then translated into a visual representation by use of locomotion models that express travel options. During the design process, the workspace analysis was iterated several times, often in combination with the visualization step. In this way, the display was designed in an incremental evolutionary way, allowing interface form and workspace analysis to give input to each other. EID is certainly not a recipe for the interface geometrical design, and a gap exists between analysis and display form. In the present context, pilots already have and use an existing natural ecology of motion through space. Since we believe that interface forms should enhance this existing ecology, it led us to look for solution forms that used the dimensions of motion and space.

This paper shows that conflict situations are, depending on conflict geometry, most efficiently resolved by a speed change, heading change, or a combination of both. Locomotion models 
such as the HTF, which do not describe combined speedheading maneuvers, fail to present travel options that minimize path deviation and support implicit coordination unless they use an explicit resolution advice. The use of heading bands or speed bands on the interface to prevent maneuvers that trigger new conflicts is unsatisfactory. The heading band only holds for the current speed and, vice versa, the speed band for the current heading. In addition, multiple conflicts may overlap each other, making it difficult to distinguish between the bands or relate individual bands with the appropriate intruder aircraft on the ND.

Separation cannot be presented in a steady meaningful way on a traditional plan view display. However, in an ownshipcentered speed-heading vector field (such as visualized in the SVE interface), separation can be expressed in terms of maneuvers based on instantaneous speed-heading changes. For each conflict, the FBZ visualizes efficient conflict resolutions (supporting conflict prevention) and intruder maneuvers (supporting implicit coordination).

Due to the time needed to maneuver, the FBZ beam width expands. Conflict urgency can be directly perceived by the expansion rate of the FBZ, a property that becomes more salient just before both aircraft cross each other. However, a pilot cannot be completely confident that it is still possible to go out of, or trespass the FBZ, as it might expand faster than the aircraft can maneuver. Therefore, a time threshold should be determined, below which collision avoidance is activated.

The mapping of the SVE on the ND (pinpointed on the ownship position geometrically) relates the aircraft in the spatial space (the ND) with the FBZ in the speed-heading space. The pilot cannot only observe the speed, heading, and relative position of aircraft in the vicinity but also can observe how these intruder variables affect the ownship's travel options. Thus, the use of speed-heading space (motion) and plan view (airspace) to present 4-D spatio-temporal constraints enhances the natural ecology that pilots have and use when moving through space. The SVE "makes visible the invisible," perfectly in line with EID principles [24].

The direct visualization of workspace constraints in the speed-heading space, the "intelligence" behind the SVE envelope mapped on the ND, allows for a deeper understanding of travel options and conflict situations. This makes it an appropriate support tool for complex problem solving. On the other hand, a pilot can simply steer out of the FBZ and remain within the speed envelope without fully analyzing and understanding all aspects of the conflict situation, reducing cognitive workload. This results in supporting the SRK levels of cognitive control.

Tentatively, the introduction of a display that enables the operator to accomplish his task with little cognitive effort might lead to an erosion of skills [26]. Extrapolating the results observed in [3] to the present context, however, suggests that if pilots actively reflect on the feedback they receive from the EID display, they will have an opportunity to gain deeper knowledge of airborne conflict situations.

A brief pilot evaluation proved that pilots are able to perform safe and efficient conflict resolution maneuvers using the display. The experiment data and pilot comments primarily served as an input for further design steps and more extensive evaluations. The limited complexity of this experiment needs to be seen in this context; it was mainly intended to elicit pilot comments and provide initial feedback on the design. From the results, it appeared that the FBZ expansion was readily observed by the pilots and that some pilots used it to form an impression of conflict urgency. FBZ expansion rate was more difficult to observe, however, leading to difficulty in predicting the development of a conflict and uncertainty in the choice and timing of maneuvers in some cases. Pilots also can correlate the FBZ lines with the associated intruder aircraft and are well able to predict how they will pass other aircraft. Movement of the FBZ is visible to the pilots, but they cannot relate that movement to the intruder maneuver causing it, unless that maneuver is a critical hostile maneuver. Pilots also indicated that they would need more practice with the display to achieve better comprehension and use it better.

\section{RECOMMENDATIONS}

The inclusion of aircraft dynamics into the travel models would make the FBZ presentation more accurate. In urgent situations, this guarantees that a given resolution is feasible. In situations where unexpected intruder behavior alters the resolution strategy, the feasibility of the trespassing maneuver is then assured. Exchange of intent information should also be explored. The presentation of autopilot settings on the SVE would enable pilots to quickly assess the intruder's intentions. One planned path change in the near future could be accounted for in the (loco)motion prediction and the presentation of the FBZ. Including both dynamics and intent information allows for more flexible travel strategies. At present, a preliminary design including these features has been made [19].

Research is ongoing to experimentally compare current airborne separation systems like P-ASAS with the ecological design, focusing on the relation between display, conflict geometry, and pilot performance and workload [1]. Since an ecological interface does not necessarily yield better performance, attention focuses on analyzing pilot problem-solving skills in exceptional situations and pilot situation awareness.

\section{REFERENCES}

[1] R. Appleton, M. Mulder, and M. M. van Paassen, "Comparison of two interfaces for supporting pilots in airborne self-separation tasks," presented at the AIAA Guidance, Navigation and Control Conf. Exhib., Keystone, CO, Aug. 2006, Paper no. AIAA-2006-6062.

[2] C. Borst, H. C. H. Suijkerbuijk, M. Mulder, and M. M. van Paassen, "Ecological interface design for terrain awareness," Int. J. Aviation Psychol., vol. 16, no. 4, pp. 375-400, Oct. 2006.

[3] K. Christoffersen, C. N. Hunter, and K. J. Vicente, "A longitudinal study of the effects of ecological interface design on deep knowledge," Int. J. Human-Comput. Stud., vol. 48, no. 6, pp. 729-762, Jun. 1998.

[4] T. Degre and X. Lefevre, "A collision avoidance system," J. Navigat., vol. 34, pp. 294-302, 1981.

[5] V. N. Duong and K. Zeghal, "Conflict resolution advisory for autonomous airborne separation in low-density airspace," in Proc. 36th Conf. Decision Control, San Diego, CA, Apr. 2003, pp. 2429-2434.

[6] "Principles of operation for the use of airborne separation assurance systems," Federal Aviation Authorities-Eurocontrol, 2001. Tech. Rep.

[7] J. J. Gibson, The Ecological Approach to Visual Perception. Boston, MA: Houghton Mifflin, 1979.

[8] J. J. Gibson and L. E. Crooks, "A theoretical-field analysis of automobiledriving," Amer. J. Psychol., vol. 51, no. 4, pp. 453-471, Jul. 1938. 
[9] J. M. Hoekstra, "Designing for safety: The free flight air traffic management concept," "Ph.D. dissertation," Delft Univ. Technol., Delft, The Netherlands, 2001.

[10] A. S. Lenart, "Collision threat parameters for a new radar display and plot technique," J. Navigat., vol. 36, no. 3, pp. 404-410, Sep. 1983.

[11] M. Lind, "Representing goals and functions of flow modelling-An introduction to multilevel flow modelling," Inst. Autom. Control Syst., Univ. Denmark, Copenhagen, Denmark, Tech. Rep. 90-D-381, 1990.

[12] Q. Liu and E. Pedersen, "Direct perception of collision danger information for safe marine navigation," in Proc. IEEE Int. Conf. Syst., Man Cybern., Hague, The Netherlands, Oct. 2004, pp. 3898-3902.

[13] M. Modarres, Functional Modelling Applications Association Website. [Online]. Available: http://www.enre.umd.edu/ifmaa/

[14] J. Rasmussen, "Skills, rules and knowledge; signals, signs and symbols, and other distinctions in human performance models," IEEE Trans. Syst., Man, Cybern., vol. SMC-13, no. 3, pp. 257-266, May/Jun. 1983.

[15] J. Rasmussen, "The role of hierarchical knowledge representation in decision making and system management," IEEE Trans. Syst., Man, Cybern., vol. SMC-15, no. 2, pp. 234-243, 1985

[16] "Airborne conflict management: Application description v2.5," Federal Aviation Authorities, Mar. 2002.

[17] "Minimal operational performance standards for traffic alert and collision avoidance system 2 (tcas2) airborne equipement," Federal Aviation Authorities, Tech. Rep., 2002.

[18] S. B. J. Van Dam, M. Mulder, and M. M. van Paassen, "Functional presentation of travel opportunities in flexible use airspace: An EID of an airborne conflict support tool," in Proc. IEEE Int. Conf. Syst., Man Cybern., Hague, The Netherlands, Oct. 2004, pp. 802-808.

[19] S. B. J. Van Dam, M. Mulder, and M. M. van Paassen, "Airborne selfseparation display with turn dynamics and intruder intent-information," in Proc. IEEE Int. Conf. Syst., Man Cybern., Montreal, QC, Canada, Oct. 2007, pp. 1445-1454.

[20] M. M. van Paassen, "Functions of space and travellers," in Proc. 18th Eur. Annu. Conf. Human Decision Making Manual Control, Oct. 1999, pp. 268-274.

[21] M. M. van Paassen and M. Mulder, "Functional and non-functional goals, design and usage of cognitive systems," in Proc. IEEE Int. Conf. Syst., Man Cybern., The Hague, The Netherlands, Oct. 2004, pp. 797-801.

[22] M. M. van Paassen and P. A. Wieringa, "Reasoning with multilevel flow models," Reliab. Eng. Syst. Saf., vol. 64, no. 2, pp. 151-165, May 1999.

[23] K. J. Vicente, "Ecological interface design: Progress and challenges," Hum. Factors, vol. 44, no. 1, pp. 62-78, 2002.

[24] K. J. Vicente and J. Rasmussen, "The ecology of human-machine systems II: Mediating direct-perception in complex work domains," Ecol. Psychol., vol. 2, no. 3, pp. 207-249, 1990.

[25] K. J. Vicente and J. Rasmussen, "Ecological interface design: Theoretical foundations," IEEE Trans. Syst., Man, Cybern., vol. 22, no. 4, pp. 589606, Jul./Aug. 1992

[26] C. D. Wickens, "Virtual reality and education," in Proc. IEEE Int. Conf. Syst., Man Cybern., Oct. 1992, vol. 1, pp. 842-847.

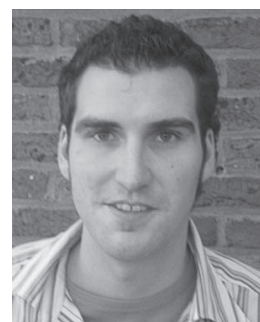

Stijn B. J. Van Dam received the M.Sc. degree in aerospace engineering from the Delft University of Technology, Delft, The Netherlands, in 2004, where he is currently working toward the Ph.D. degree. His $\mathrm{Ph}$.D. work concentrates on the design and validation of an ecological self-separation assistance display in cruise flight. The research presented in this paper is part of his thesis.

$\mathrm{He}$ is also working as a Researcher with the Aerospace Software and Technologies Institute, Faculty of Aerospace Engineering, Delft University of Technology. His research includes the design of ecological human-machine interfaces that support for vehicle control and traffic management in the aviation and maritime domains.

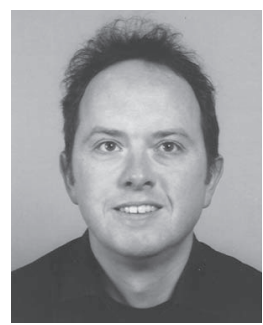

Max Mulder received the M.Sc. and Ph.D. degrees (cum laude) in aerospace engineering from the Delft University of Technology, Delft, The Netherlands, in 1992 and 1999, respectively, for his work on the cybernetics of tunnel-in-the-sky displays.

$\mathrm{He}$ is currently an Associate Professor with the Control and Simulation Division, Faculty of Aerospace Engineering, Delft University of Technology. His research interests include cybernetics and its use in modeling human perception and performance, and cognitive systems engineering and its application in the design of "ecological" human-machine interfaces.

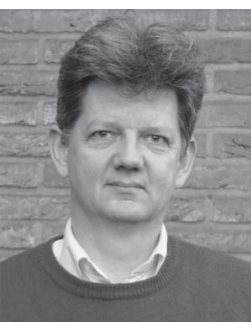

M. M. van Paassen received the M.Sc. degree from the Delft University of Technology, Delft, The Netherlands, in 1988, where he also received the $\mathrm{Ph} . \mathrm{D}$. degree in 1994, with a thesis about a neuromuscular system of the pilot's arm, for studying manual control of aircraft.

After this, he spent two years as a Brite/EuRam Research Fellow with the University of Kassel, Kassel, Germany, with Prof. Johannsen, where he worked on the development of alternative interfaces for process control based on functional models of the process. During a subsequent stay with the Technical University of Denmark, he put the practical experience gained in Kassel to use in theoretical work on multilevel flow modeling. Currently, he is with the Faculty of Aerospace Engineering, Delft University of Technology, working on aircraft simulation and human-machine interaction. 